FORMATION Formation emploi

Revue française de sciences sociales

109 | janvier-mars 2010

L'orientation scolaire et professionnelle dans un monde incertain

\title{
Orientation et affectation : la sélection dans l'enseignement professionnel du second degré
}

Guidance and allocation: selection processes in vocational secondary education Orientierung und Verwendung: Selektion im beruflichen Zweig der weiterführenden Schule

Orientación y asignación : la selección en la enseñanza profesional de segundo grado

Jean-Jacques Arrighi et Céline Gasquet

\section{OpenEdition}

Journals

Édition électronique

URL : http://journals.openedition.org/formationemploi/2814

DOI : $10.4000 /$ formationemploi.2814

ISSN : 2107-0946

Éditeur

La Documentation française

Édition imprimée

Date de publication : 15 mars 2010

Pagination : $99-112$

ISSN : 0759-6340

Référence électronique

Jean-Jacques Arrighi et Céline Gasquet, « Orientation et affectation : la sélection dans l'enseignement professionnel du second degré », Formation emploi [En ligne], 109 I janvier-mars 2010, mis en ligne le 01 mars 2012, consulté le 30 octobre 2020. URL : http://journals.openedition.org/formationemploi/ 2814 ; DOI : https://doi.org/10.4000/formationemploi.2814 


\section{DOSSIER}

\section{Orientation et affectation : la sélection dans l'enseignement professionnel du second degré}

Jean-Jacques Arrighi et Céline Gasquet*

La réflexion sur l'orientation ignore trop souvent les réalités des processus d'affectation. Au-delà des aspirations des jeunes, conditionnées par leur histoire familiale, leur orientation résulte d'un univers de contraintes liées à la rareté des spécialités de formation sur un territoire donné, à la nature fortement sexuée de ces formations et à l'existence de discriminations.

«Ses grandes boucles d'oreilles conductrices d'électricité encerclaient la moitié des joues ».

"Non, c'était pour l'orientation. L'autre jour, j'ai visité le lycée Marcel-Aymé. M'sieur, c'est pas possible, j’vais pas pouvoir y aller, y'a qu'des gothiques là-bas. »

Entre les murs, film de François Begaudeau.

Au sein du cadre plus vaste de la formation tout au long de la vie, la question d'une réforme indispensable des services en charge de l'orientation scolaire alimente un débat récurrent, particulièrement actif depuis quelques années ${ }^{1}$. Au-delà des questions réelles

\footnotetext{
1 En 2004, le Haut Conseil de l’Évaluation de l'École a rendu un avis sur l'orientation à la fin du collège et du lycée (http://cisad. adc.education.fr/hcee/documents/avis12.pdf) ; en 2005, les deux Inspections générales de l’Éducation nationale ont rédigé un rapport contenant une vingtaine de propositions (http://www.education. gouv.fr/cid2223/le-fonctionnement-des-services-d-information-etd-orientation.html) ; en 2007, un autre rapport a été établi par le député Frédérick Weiss (http://www.debats-parlementaires.fr/12/
}

* Jean Jacques Arrighi est chargée d'études au Céreq, département "Entrées et évolution dans la vie active ". Ses travaux portent sur l'insertion professionnelle des jeunes sortants de l'enseignement secondaire, sur le développement des formations en alternance et sur l'évolution des systèmes de formation professionnelle en région. Parmi ses dernières publications figurent Arrighi J.J., Brochier D. (2009), "L'apprentissage au sein de l'Éducation nationale: une filière sortie de la clandestinité ", Céreq, Notes Emploi Formation, n 40 
Arrighi J.J. (coord.) (2007), Géographie de la formation professionnelle, Paris, Conseil national de la formation professionnelle tout au long de la vie (CNFPTLV), 86 p.

Céline Gasquet est chargée d'études au Céreq, département "Entrées et évolution dans la vie active ". Ses travaux portent sur l'insertion professionnelle des jeunes sortant de l'enseignement secondaire et des jeunes résidant dans des quartiers défavorisés, cibles de la politique de la ville, ainsi que sur la dimension territoriale de l'insertion des jeunes et l'évolution des systèmes de formation professionnelle en région. Parmi ses dernières publications figure: Couppié T., Gasquet C. (2009), "Quartiers défavorisés : relégation pour certains jeunes, insertion sociale et professionnelle pour d'autres », Céreq Brefn n 26, février.

Ils ont publié conjointement : Arrighi J.J., Gasquet C. et Joseph O. (2009), "Qui sort de l'enseignement secondaire? Origine sociale, parcours scolaires et orientation des jeunes de la Génération 2004 ", Céreq Note Emploi Formation, $n^{\circ} 41$, juin. Arrighi J.J., Gasquet C. et Joseph O. (2009), "L'insertion des sortants de l'enseignement secondaire. Des résultats issus de l'enquête Génération 2004 », Céreq, Note Emploi Formation $n^{\circ} 42$, juin.

que pose la gouvernance protéiforme d'un ensemble incertain d'acteurs intervenant dans le domaine de l'Accueil, Information, Orientation (AIO) - sur des territoires tout aussi incertains et à destination de publics qui restent cloisonnés (Borras I., 2008) - une opinion semble partagée par les nombreux protagonistes: l'orientation scolaire fonctionne mal. Insuffisamment attentive aux réalités de l'économie et du marché du travail, et trop influencée par une approche de la réalisation de soi issue de la psychologie, elle contribuerait à conduire les jeunes dans l'impasse des professions sans débouchés. Elle serait largement responsable du décalage entre les qualifications détenues par les jeunes et les emplois qu'ils sont susceptibles d'occuper.

rapports/r2085.asp) et en 2008, le Haut Conseil de l'Éducation a publié un nouveau rapport.
Pourtant, la parole donnée aux élèves (Berthet, Dechezelles, Gouin, Simon, 2008) révèle des processus plus complexes, au sein desquels le rôle des agents spécialisés que sont les conseillers d'orientation psychologues (COP) est limité. Et, plus généralement, il apparaît que le vécu des élèves invalide, parmi d'autres, cette idée reçue: «les préjugés les plus courants s'opposent de manière relativement importante à l'opinion que se font les élèves du rôle, de l'importance et des modalités de l'orientation ». En 2008, le Haut Conseil de l'Éducation² souligne également la multiplicité des processus et précise clairement la dualité des enjeux : « Le terme "orientation” recouvre deux activités que la langue anglaise distingue: le processus qui répartit les élèves dans les différentes voies de formation, filières et options ("student distribution ») et l'aide aux individus dans le choix de leur avenir scolaire et professionnel (« vocationnal guidance », "school and carreer conselling»)». De ces deux dimensions, il apparaît clairement que la première est d'une toute autre importance que la seconde: "C'est l'offre de formation qui régit les politiques d'orientation dans les académies. Du point de vue de l'Éducation nationale, une rentrée scolaire est considérée comme réussie lorsque chaque élève a une place, quels qu'aient été ses vœux, et que chaque professeur est devant une classe. Le nombre de places disponibles par filière fait partie des contraintes qui pèsent sur l'orientation des élèves ${ }^{3}$. Force est de constater que, de ce point de vue, l'orientation scolaire fonctionne. Chaque année, les générations d'élèves sont triées et les structures pédagogiques existantes sont alimentées.

Focalisé sur les scolarités des jeunes qui entrent dans la vie active à l'issue de l'enseignement secondaire, l'objet de ce bref article ${ }^{4}$ est de montrer qu'au final la place de chaque élève doit peu au hasard. Prolongeant la hiérarchie enseignement général, puis technologique puis professionnel, les spécialités de formation et les filières (apprentissage-voie scolaire) organisent un système classant où les jeunes les moins bien informés

\footnotetext{
2 « Bilan des résultats de l'École - 2008-L'orientation scolaire », rapport : http://www.hce.education.fr

3 « Bilan des résultats de l'École - 2008-L'orientation scolaire », (p. 19).

${ }^{4}$ Cet article reprend, en les problématisant, les principaux résultats détaillés dans la NEF-Céreq $n^{\circ}$ 41, (Arrighi, Gasquet, Joseph, 2009a).
} 


\section{Encadré 1 \\ Méthodologie 1}

Au printemps 2007, le Céreq a interrogé un échantillon national d'environ 34000 individus sortis de formation initiale, au cours de l'année scolaire 2003-2004. Ces individus sont issus de tous les niveaux de formation et sont représentatifs des 737000 jeunes qui, cette année-là, ont quitté pour la première fois le système éducatif. Cette enquête, qui s'inscrit dans le dispositif des enquêtes générationnelles du Céreq, succède aux trois précédentes réalisées auprès des jeunes sortis de formation initiale en 1992, en 1998 et en 2001 . Son objectif est d'analyser les premières années de vie active en relation avec les caractéristiques sociales et les parcours scolaires des jeunes.

Le travail présenté dans cet article s'appuie sur l'analyse de la population des jeunes de cette génération 2004 qui sont sortis de l'enseignement secondaire sans avoir accédé à l'enseignement supérieur au cours de leur formation initiale. L'enquête permet de situer l'origine sociale et nationale des individus et la situation de leurs parents dans le système d'emploi. Elle permet en outre de reconstituer leurs trajectoires scolaires et les conditions de leur orientation. Enfin, elle recueille les motifs qu'ils avancent pour expliquer l'interruption de leurs études.

ou dotés se trouvent relégués dans des formations qui, bien que sans perspectives professionnelles très claires, existent ou dont le maintien est peu coûteux.

À l'appui de cette hypothèse, nous mobilisons les résultats de l'enquête Génération 2004 (cf. encadré méthodologique 1) du Céreq qui, pour une cohorte de sortants du système éducatif, permet de recouper l'origine sociale et nationale des jeunes, leur orientation en troisième, le niveau du diplôme détenu lorsqu'ils entrent sur le marché du travail et les raisons qu'ils invoquent pour expliquer l'interruption de leurs études. Nous organiserons notre propos en trois points. Le premier montrera la fragilité de deux idées convenues, communément admises : la réticence des jeunes à l'égard de l'apprentissage et leur appétence pour des spécialités sans débouchés. Le second rappellera l'importance de certaines rigidités trop souvent négligées, comme les discriminations à l'œuvre au sein de l'apprentissage et la ségrégation sexuée qui gouverne l'orientation dans les différentes spécialités de formation. Le troisième proposera un regroupement en cinq ensembles des spécialités de formation et révèlera l'hétérogénéité et la hiérarchisation de l'enseignement professionnel dans le second degré. Au terme de cette synthèse, l'enjeu d'une réforme qui ne porterait que sur l'organisation de l'orientation scolaire apparaîtra limité.

\section{DEUX IDÉES REÇUES INVALIDÉES}

Première idée reçue : faute d'une information suffisante, les élèves ne choisiraient pas d'entrer en apprentissage, alors que celui-ci améliore considérablement l'insertion dans le système d'emploi - ce qui est vrai. Seconde idée reçue : les élèves plébisciteraient en revanche les formations tertiaires du secrétariat et de la comptabilité, au détriment des formations conduisant à de nombreux emplois disponibles, comme celles du bâtiment ou de l'hôtellerie. Pour la plupart des acteurs du monde politique ou économique, ces propositions sont si certaines qu'elles ne méritent pas d’être vérifiées.

Néanmoins, pour les générations actuelles, elles sont fausses.

Pour les garçons comme pour les filles de la Génération 2004, le choix de l'apprentissage apparaît plus souvent comme le premier vœu exprimé. Pour 85 \% des garçons entrés en apprentissage en seconde CAP (certificat d'aptitude professionnelle), il s'agissait de leur premier vœu ; en regard, ce n'était le cas que pour $72 \%$ des garçons entrés dans des classes identiques en lycée professionnel. Le constat est similaire pour les garçons entrés en BEP (brevet d'études professionnelles). Et il en va de même pour les filles entrées en première année de CAP ou de BEP. Ce résultat rejoint les observations issues du terrain, dont les dernières en date réalisées dans les Pays de la Loire : « Le choix de l'apprentissage est de plus en plus vécu comme un choix personnel. $79 \%$ des apprentis ligériens 
Tableau 1

Orientation à l'issue de la troisième

\begin{tabular}{|l|c|c|}
\hline \multicolumn{1}{|c|}{ \% de premier vœu parmi les jeunes entrés en : } & Garçons & Filles \\
\hline Première année de BEP apprentissage & 85 & 73 \\
\hline Première année de BEP scolaire & 75 & 70 \\
\hline Première année de CAP apprentissage & 85 & 80 \\
\hline Première année de CAP scolaire & 72 & 68 \\
\hline
\end{tabular}

Source : Enquête Génération 2004, Céreq.

Champ : sortants de l'enseignement secondaire.

Sigles : CAP : certificat d'aptitude professionnelle. BEP : brevet d'études professionnelles.

justifient ainsi leur entrée dans l'apprentissage. Ils n'étaient que 62,7 \% en 1992 » (Moreau, 2008b).

Par ailleurs, les jeunes qui se déclarent insatisfaits de leur orientation à l'issue de la troisième n'auraient pas tous voulu poursuivre dans la voie générale. Les préférences exprimées dans les réponses à une question posée aux individus dont le premier vœu n'avait pas été satisfait le démontrent. Pour les garçons comme pour les filles, entre 20 et $25 \%$ auraient préféré une seconde générale, entre 38 et $49 \%$ auraient choisi une autre spécialité de formation et entre 16 et $28 \%$ voulaient entrer en apprentissage ${ }^{5}$. À ce sujet, le rapport du Haut Comité à l’Éducation remarque : "l'élève orienté en lycée professionnel peut se voir affecté dans une spécialité qui ne l'intéresse pas ou qui ne correspond pas à ses aptitudes, ou bien il peut être contraint de quitter son secteur géographique, ou les deux: c'est là une différence importante avec le lycée général et technologique où, sauf pour certaines options à recrutement limité, l'offre de seconde est adaptée constamment aux effectifs et uniformément répartie sur le territoire. Malgré la mauvaise image de l'enseignement professionnel, il est plus difficile d'intégrer certaines de ses spécialités que d'entrer dans une seconde générale ».

Or, lorsque l'on observe la satisfaction du premier vœu d'orientation, selon la spécialité de formation de la classe de sortie, les écarts peuvent approcher les trente points. Plus de $80 \%$ des sortants des formations du domaine de la santé, de la coiffure-esthétique,

${ }^{5}$ On retrouve un résultat identique dans l'exploitation du Panel 1995 de la DEPP : «les refus de vœu ont concerné principalement la spécialité de formation et plus rarement le choix de la seconde » (Boudesseul G., Grelet Y., 2008, p. 30). des spécialités agricole, du sanitaire et social, de la mécanique auto, des transports et du travail du bois avaient été orientés en fin de troisième conformément à leur premier vœu. Ce n'est le cas que pour moins de $60 \%$ des sortants des spécialités de l'habillement, des textiles et du cuir, des services à la collectivité, de la comptabilité ou du secrétariat. Les spécialités de formation conduisant vers les métiers dits en tension, réputées rebutantes pour les jeunes, comme par exemple celles de l'alimentation, du bâtiment ou de l'hôtellerie, sont en réalité « arbitrées »6 et exprimées en premier vœu à la sortie de la troisième dans plus de sept cas sur dix; quand, dans le même temps, les spécialités du tertiaire administratif comme le secrétariat ou la comptabilité - où les jeunes se précipiteraient en dépit d'un avenir professionnel très hypothétique - ne sont choisies en premier vœu à la fin de la troisième que par un peu plus d'un élève sur deux parmi ceux qui s’y retrouvent.

Ainsi, d'une part, l'apprentissage ne manque pas de candidats, mais manquerait plutôt de places, et, d'autre part, au moins $40 \%$ des jeunes orientés vers les spécialités de formation du tertiaire administratif ne l'avaient pas souhaité (comptabilité, gestion ou secrétariat-bureautique, $c f$. Tableau 2). Or, on sait depuis longtemps (Grelet, Viney, 1991) que ces spécialités souffrent d'une absence de reconnaissance aux premiers niveaux de qualification.

Pour autant, les « orientations frustrées » n’ont pas été distribuées au hasard. Deux phénomènes ont en particulier joué, qui sont massifs et dont on parle peu.

\footnotetext{
${ }^{6}$ Les jeunes font un arbitrage entre leurs souhaits les plus fous et la réalité à laquelle ils doivent s'adapter.
} 
Tableau 2

Satisfaction du premier vœu d'orientation (en \%)

\begin{tabular}{|c|c|c|}
\hline \multicolumn{3}{|c|}{ « Votre orientation à l'issue de la troisième correspondait-elle à votre premier vœu? » } \\
\hline Spécialité de formation de la classe de sortie & oui & non \\
\hline Habillement, textile, cuir & 50 & 50 \\
\hline Spécialités des services à la collectivité & 52 & 48 \\
\hline Comptabilité, gestion & 55 & 45 \\
\hline Secrétariat, bureautique & 58 & 42 \\
\hline Toutes spécialités des transformations & 62 & 38 \\
\hline Mécanique de précision, y compris aéronautique & 70 & 30 \\
\hline Travail social & 71 & 29 \\
\hline Structures métalliques & 71 & 29 \\
\hline Commerce, vente & 72 & 28 \\
\hline Bâtiment : finitions & 74 & 26 \\
\hline Génie civil, mines, travaux publics & 76 & 24 \\
\hline Bâtiment / construction et couverture & 76 & 24 \\
\hline Électricité, électronique & 77 & 23 \\
\hline Accueil, hôtellerie, tourisme & 78 & 22 \\
\hline Énergie, génie climatique & 79 & 21 \\
\hline Spécialités pluri-technologiques mécaniques-électricité & 80 & 20 \\
\hline Agro-alimentaire, alimentation, cuisine & 80 & 20 \\
\hline Travail du bois et de l'ameublement & 83 & 17 \\
\hline Transport, manutention, magasinage & 83 & 17 \\
\hline Moteurs et mécanique auto & 84 & 16 \\
\hline Spécialités plurivalentes sanitaires et sociales & 86 & 14 \\
\hline Spécialités agriculture, élevage, paysage, forêts & 86 & 14 \\
\hline Coiffure, esthétique et autres spécialités des services aux personnes & 86 & 14 \\
\hline Santé & 88 & 12 \\
\hline
\end{tabular}

Source : Enquête Génération 2004. Céreq

Champ : sortants de l'enseignement secondaire.

La question - posée uniquement aux jeunes orientés vers l'enseignement professionnel à l'issue de la troisième - était : "Cette orientation correspondait-elle à votre premier vœu ? ", de manière à correspondre exactement à la formulation utilisée au cours des procédures d'orientation, et donc à limiter les effets bien connus de reconstruction biographique dans les enquêtes rétrospectives. De fait, les enquêtes Génération surestiment légèrement la proportion de premiers vœux satisfaits qui, pour les jeunes orientés en CAP ou en BEP (certificat d'aptitude professionnelle et brevet d'études professionnelles), est de l'ordre de $70 \%$ dans le Panel 95 de la DEPP, panel dans lequel la même question était posée (Boudesseul G., Grelet Y., 2008, p. 34).

Note de lecture : $50 \%$ des jeunes qui sortent de l'enseignement secondaire dans la spécialité « habillement, textile, cuir » ont déclaré que leur orientation à l’issue de la 3 e correspondait à leur premier vœu. 


\section{DEUX RIGIDITÉS MAJEURES IGNORÉES DANS LES DÉBATS}

La première de ces rigidités est le caractère fortement sexué de l'enseignement technologique et professionnel dans le second degré. L'enseignement général est mixte. L'enseignement technologique, et plus encore l'enseignement professionnel le sont peu. Quelquefois pas du tout.
Parmi les sortants de l'enseignement secondaire de la Génération 2004, 33 \% sortent de spécialités quasi exclusivement masculines. Les filles représentent ainsi moins de $5 \%$ des jeunes entrant dans la vie active à l'issue d'une formation des spécialités de la métallerie, du bâtiment, de la mécanique auto, de l'électricité-électronique, du bois, de l'énergie et du génie civil. Spécialités qui, d'une façon générale, connaissent des difficultés d'insertion moindres à l'entrée sur le marché du travail. À l’opposé, les garçons ne

Tableau 3

Ségrégation sexuelle dans les spécialités de l'enseignement professionnel

\begin{tabular}{|l|c|c|c|}
\hline \multicolumn{1}{|c|}{ Spécialité de formation à la sortie du système éducatif } & \% d'hommes & \% de femmes & Effectifs de sortants \\
\hline $\begin{array}{l}\text { Coiffure, esthétique et autres spécialités des services } \\
\text { aux personnes }\end{array}$ & 4 & 96 & 8500 \\
\hline Secrétariat, bureautique & 5 & 95 & 16540 \\
\hline Santé & 6 & 94 & 16870 \\
\hline Habillement, textile, cuir & 9 & 91 & 6170 \\
\hline Spécialités plurivalentes sanitaires et sociales & 12 & 88 & 17330 \\
\hline Travail social & 21 & 79 & 2450 \\
\hline Spécialités des services à la collectivité & 26 & 74 & 3820 \\
\hline Commerce, vente & 34 & 66 & 30120 \\
\hline Spécialités plurivalentes des échanges et de la gestion & 40 & 60 & 20100 \\
\hline Accueil, hôtellerie, tourisme & 42 & 58 & 15240 \\
\hline Comptabilité, gestion & 48 & 52 & 13620 \\
\hline Toutes spécialités des transformations & 64 & 36 & 4130 \\
\hline Agro-alimentaire, alimentation, cuisine & 75 & 25 & 15710 \\
\hline Spécialités agriculture, élevage, paysage, forêts & 79 & 21 & 15230 \\
\hline Transport, manutention, magasinage & 82 & 18 & 4350 \\
\hline Génie civil, mines, travaux publics & 96 & 4 & 4490 \\
\hline Mécanique de précision, y compris aéronautique & 97 & 3 & 13660 \\
\hline Énergie, génie climatique & 97 & 3 & 6110 \\
\hline Travail du bois et de l'ameublement & 98 & 2 & 10920 \\
\hline Spécialités pluri-technologiques mécaniques-électricité & 98 & 2 & 9970 \\
\hline Électricité, électronique & 98 & 2 & 25030 \\
\hline Bâtiment : construction et couverture & 99 & 1 & 7130 \\
\hline Moteurs et mécanique auto & 99 & 1 & 16220 \\
\hline Bâtiment : finitions & 99 & 10170 \\
\hline Structures métalliques & 99 & 13000 \\
\hline
\end{tabular}

Note de lecture : parmi les 16540 jeunes sortants de l'enseignement professionnel secondaire, en 2004, dans la spécialité secrétariatbureautique, $5 \%$ sont des hommes et $95 \%$ des femmes.

Source : Enquête Génération 2004, Céreq. 
sont représentés de façon marginale (moins de $10 \%$ ) que dans quatre spécialités, qui de plus ne rassemblent que $14 \%$ des effectifs de sortants : la coiffure esthétique, la santé, le secrétariat et le textile-habillement. La diversité des choix possibles est donc beaucoup plus riche pour les garçons que pour les filles. La séparation des sexes est, on le sait, une caractéristique prégnante de l'âge adolescent au cours duquel l'orientation de fin de troisième se déroule. Dès lors, à l'exception de quelques projets professionnels bien trempés, les filles, écartées de leur plein gré de nombreux établissements d'enseignement et de CFA (centre de formation d'apprentis), se rassemblent en nombre dans les formations générales, et dans les formations technologiques et professionnelles de l'hôtellerie, du commerce et du tertiaire administratif qui, hormis le secrétariat, sont mixtes, ou dans les spécialités très féminisées de la santé et du champ sanitaire et social.

La seconde rigidité est très discrète. Il est vrai qu'elle est embarrassante : l'apprentissage est sélectif et cette sélection n'est pas neutre du point de vue des origines nationales (Moreau, 2008a), sociales et territoriales. La séparation des sexes est encore plus prononcée dans l'apprentissage que dans l'enseignement professionnel. La répartition par sexe et spécia- lité de formation des effectifs inscrits dans les CFA du secondaire le montre clairement, nous n'y reviendrons pas. L'enquête Génération 2004 permet en revanche de mettre en évidence une sélection des publics sur critères ethniques. Elle montre également que la situation d'activité des parents a une incidence certaine, et que la sélection des publics s'inscrit dans le cadre d'une offre inégalement répartie sur le territoire.

En ce qui concerne les origines nationales, l'enquête nous livre deux indices robustes de l'existence de discriminations. Un quart des jeunes issus de l'enseignement secondaire ont au moins un de leurs parents né à l'étranger, cette proportion atteint $35 \%$ pour les sortants de collège et elle est de $20 \%$ pour les jeunes quittant l'école après un bac général. Or, elle n’est que de $12 \%$ pour les sortants d'un brevet professionnel, diplôme qui ne se prépare qu'en apprentissage. Et si l'on cerne plus précisément les individus susceptibles d'être discriminés ${ }^{7}$ pour des raisons liées à leur origine

\footnotetext{
7 Les mécanismes auto-entretenus de ces discriminations sont décrits dans un rapport commandé par l'ACFCI (Assemblée des chambres françaises de commerce et d'industrie) dont certains résultats sont parus dans la presse, sans qu'il ne soit jamais officiellement publié ( $c f$. dépêche Agence Emploi Formation (AEF) n 60860 du 31 janvier 2006: «Les discriminations raciales
}

Filière de formation et pays de naissance des parents

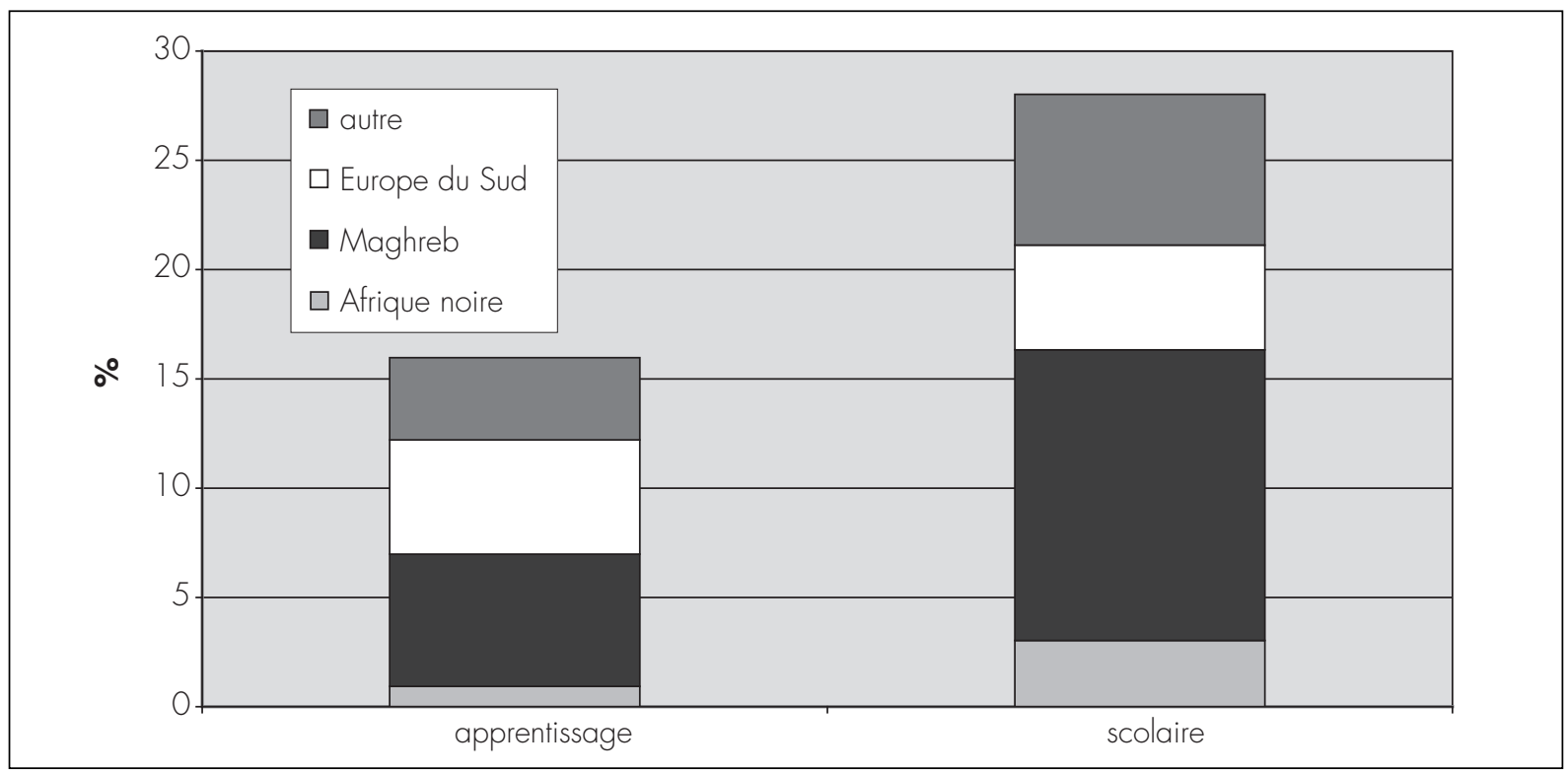

Source : Enquête Génération 2004. Céreq

Champ : sortants du secondaire. 
ou à leur couleur de peau, les enfants de parents nés au Maghreb ou en Afrique noire sont en proportion deux fois plus rares parmi les sortants d'apprentissage ${ }^{8}$ que parmi les jeunes issus de l'enseignement professionnel scolaire (cf. Graphique 1) ( $c f$. Farvaque, 2009). De ce point de vue, l'apprentissage importe, dans le système éducatif, les discriminations à l'œuvre sur le marché du travail. Ainsi, les spécialités de formation où l'apprentissage est très présent, l'alimentation, le bâtiment gros œuvre, la coiffure, la mécanique automobile et le travail du bois sont celles où la part des jeunes dont l'un au moins des parents est né au Maghreb est la plus faible.

L'apprentissage est également peu ouvert aux enfants de chômeurs ou d'inactifs. Parmi les jeunes dont le père travaillait, les différences d'origine sociale sont faibles entre jeune apprenti et jeune lycéen. Seule une légère surreprésentation des parents agriculteurs ou artisans au bénéfice des apprentis apparaît. Pour le reste, dans la majorité des cas, le père était ouvrier (36\%) ou employé (25\%). En revanche, l'absence d'emploi chez le père et la mère est presque deux fois plus fréquente pour les scolaires (11\%) que pour les apprentis (6 \%). Enfin, les formations par apprentissage sont beaucoup plus présentes en milieu rural. Les jeunes issus de zones rurales ${ }^{9}$ représentent ainsi $24 \%$ des sorties de l'apprentissage au niveau secondaire et seulement $19 \%$ des sorties de l'enseignement scolaire. En lien avec cette spécificité des filières de formation, la part de jeunes issus du milieu rural, en 2004, varie de moins de $17 \%$ dans les spécialités des transformations industrielles, l'habillement, le secrétariat, ou encore l'électricité, où l'apprentissage est peu présent, à $25 \%$ et plus dans les spécialités du transport, de l'hôtellerie, du bois et de l'agriculture. Et si la proportion de jeunes domiciliés dans une zone

n'épargnent pas les apprentis des CFA des chambres de commerce et d'industrie, selon une étude réalisée pour l'ACFCI »).

8 À cet égard, un fait mérite d'être souligné : parmi les sortants de la Génération 2004, le contrat de professionnalisation est systématiquement plus ouvert que le contrat d'apprentissage, à une certaine diversité d'origine, c'est vrai en particulier pour les jeunes dont l'un au moins des parents est né en Afrique noire ou au Maghreb. Ces derniers représentent $15 \%$ des entrées de jeunes non diplômés en contrat de professionnalisation contre $7 \%$ seulement des entrées en apprentissage, et, respectivement, $14 \%$ contre $6 \%$ des entrées de titulaires de CAP/BEP ou encore $13 \%$ contre $7 \%$ des entrées de bacheliers. Il semble ainsi qu'aux discriminations du marché du travail s'en ajoutent certaines qui relèvent des CFA eux-mêmes. Le phénomène est décrit dans le rapport à l'AFCI cité plus haut.

9 Selon le Zonage en Aires Urbaines (ZAU) de l'INSEE. rurale est plutôt plus faible chez les sortants de collège ou de lycée non diplômés, elle atteint 27 \% pour les sortants d'une préparation au brevet professionnel.

Dans la réalité, ces rigidités liées au sexe, à l’origine sociale, nationale et territoriale, se combinent au niveau scolaire à la fin du collège et contribuent à déterminer un univers des possibles pour chaque individu qui vit plus ou moins bien son « orientation » et son entrée dans la vie active. La combinaison de ces différentes dimensions dessine un enseignement professionnel du second degré fracturé et hiérarchisé où, selon le dicton, « qui se ressemble s'assemble ».

\section{QUATRE BLOCS DE LOGIQUES}

Réduisant la focale uniquement sur les sortants de l'enseignement professionnel ${ }^{10}$, une très forte hétérogénéité distingue en effet les spécialités de formation. L'enseignement professionnel se révèle profondément composite au regard des niveaux de sortie (CAP, BAC, sorties en cours de cycle, sorties sans diplômes), des publics accueillis, des conditions d'orientation et des motifs d'arrêt études. Le choix - ou le non choix d'une spécialité de formation influence la probabilité de sortie à un certain niveau, et, dans certaines conditions, d'accès au marché du travail ; ce choix est lui-même fortement influencé par le sexe, l’origine sociale, l'origine nationale et le lieu de résidence au collège. De ce point de vue, l'analyse conduite parmi les sortants de génération 2004 rejoint les résultats d'une précédente recherche (Grelet, 2005), menée à partir de l'orientation de 18000 élèves entrés en sixième en 1995.

Pour autant, un certain nombre de similitudes (cf. encadré 2) réunissent des ensembles de spécialités, dessinant ainsi les contours d'une segmentation analytique. Cinq groupes se distinguent par des caractéristiques partagées qui leurs confèrent des profils communs. Le cinquième et dernier groupe cumule tous les handicaps.

\footnotetext{
10 Nous excluons ici de l'analyse les jeunes sortis de l'enseignement technologique. En effet, la plupart d'entre eux poursuit ses études dans l'enseignement supérieur et la population que nous observons (les sortants de l'enseignement secondaire) ne peut être représentative de leur parcours et de leurs choix.
} 


\section{Encadré 2}

\section{Méthodologie 2}

La typologie esquissée sur le champ de l'enseignement professionnel est le résultat d'une classification ascendante hiérarchique (CAH) utilisant les variables suivantes : la part de l'apprentissage, les proportions de jeunes sortis "sans qualification", sortis sans diplômes d'une terminale CAP ou BEP, diplômés du CAP ou du BEP, sortis en première professionnelle, sortis sans diplômes d'une terminale générale technologique ou professionnelle, ou, enfin, diplômés d'un bac ou d'un BP ; la part de jeunes déclarant avoir arrêté leurs études parce qu'ils avaient trouvé un emploi et la part de ceux qui déclaraient avoir atteint le niveau souhaité ; la proportion de jeunes dont l'orientation correspond à leur premier vœu, la part de jeunes n'ayant pas suivi de classe de 3 e ; le sexe ; la part de jeunes ayant deux parents sans emploi en 2004, ayant deux parents en emploi en 2004 ; la proportion de ceux dont le père était ouvrier, était employé, était artisan, était cadre; la part des jeunes dont les deux parents sont nés en France, dont l'un au moins des parents est né en Afrique noire, est né dans un pays du Maghreb, est né en Europe du Sud; et enfin les jeunes habitant en zone rurale en be et la part de ceux dont le domicile était en zone urbaine.

Sigles : CAP : certificat d'aptitude professionnelle. BEP : brevet d'études professionnelles ; BP : brevet professionnel.

Deux groupes relèvent de la reproduction des métiers de proximité, assez clairement identifiés et souvent exercés dans le cadre de l'artisanat ou de la petite entreprise.

Le premier rassemble les formations aux métiers du bâtiment, du travail du bois et du métal, et celles préparant aux métiers de l'alimentation (boucherie, charcuterie, boulangerie, poissonnerie, etc.). Ces spécialités, très fortement masculines, où l'apprentissage est majoritaire - entre 50 et $75 \%$ des jeunes sortent d'un CFA -, sont caractérisées par la faiblesse des sorties au niveau du baccalauréat ou en première professionnelle, et par l'accueil d'une forte proportion de jeunes ayant connu des difficultés scolaires au collège. Les jeunes qui en sont issus sont, en proportion relative, nombreux à considérer qu'ils ont atteint le niveau souhaité et ils sortent plus souvent qu'en moyenne parce qu'ils ont trouvé un emploi.

Le second groupe rassemble les formations aux métiers de la coiffure et de l'esthétique, des transports et de la logistique, de l'agriculture, de la réparation automobile, de l'hôtellerie et du champ sanitaire et social. Ces spécialités, où l'apprentissage est également très présent, sont caractérisées par leur très forte attractivité pour les jeunes qui, à l'exception de l'hôtellerie, les ont choisies en premier vœu dans plus de
$85 \%$ des cas. Elles scolarisent plus fréquemment que les autres les enfants d'ouvriers et d'employés dont les deux parents sont nés en France. Les jeunes dont l'un au moins des parents est né au Maghreb ou en Afrique noire y sont en proportion beaucoup moins nombreux qu'ailleurs et - toujours à l'exception de l'hôtellerie les jeunes de ces spécialités ont fréquemment leurs deux parents qui travaillent. Enfin, les jeunes d'origine rurale y sont surreprésentés.

La cible métier est plus large pour les jeunes qui se rassemblent dans le troisième groupe, constitué des spécialités plus généralistes du commerce et de l'industrie : l'électricité, l'électronique, la mécanique de précision, le génie civil et le génie climatique. Il se caractérise par l'importance des poursuites d'études après le BEP et, en corollaire, des sorties au niveau du bac ou en première professionnelle très fréquentes. La logique des enseignements disciplinaires domine ici la logique métier. Le niveau atteint importe. En dehors des formations aux métiers du commerce, ce groupe est exclusivement masculin ; pour le reste, il est assez hétérogène, tant du point de vue des conditions d'entrée dans la vie active que de l'orientation ou de l'origine sociale.

Le quatrième groupe, constitué des formations terminales aux métiers de la santé et du travail social, vise le 
plus souvent à l'exercice de professions règlementées. Les individus issus de ces formations, très majoritairement de sexe féminin, sortent le plus souvent avec un diplôme de niveau $\mathrm{V}$ (aide soignante, auxiliaire de puériculture, auxiliaire de vie sociale), en ayant atteint le niveau souhaité et en ayant trouvé un emploi. Les plus âgés des sortants de l'enseignement secondaire (plus de 21 ans en moyenne) concluent ici une formation initiale, qui a pu être assez diverse, par une formation très spécialisée, le plus souvent rationnée (le nombre de place est limité par une programmation nationale). Les filles dont au moins un des parents est né en Afrique noire y sont surreprésentées, de même que les enfants de cadre et d'employé.

\section{ET UN BLOC DE PROBLÈMES}

Le cinquième et dernier groupe réunit les spécialités qui conduisent aux métiers de la comptabilité et du secrétariat, les spécialités peu attractives des services à la collectivité (sécurité, nettoyage) ou des industries de premières transformations (conduite de chaînes automatisées) et les spécialités préparant à l'avenir incertain des métiers du textile et de l'habillement. L'éloignement de l'emploi et l'insatisfaction vis-à-vis de l'orientation caractérisent cet univers. Les jeunes y sont en proportion beaucoup plus nombreux à n'avoir aucun parent en emploi et beaucoup moins nombreux à interrompre leurs études parce qu'ils ont trouvé un emploi. Plus souvent que dans tous les autres groupes, leur orientation n'a pas été conforme à leur premier vœu, et plus souvent que dans tous les autres groupes, ils n'avaient pas atteint le niveau qu'ils auraient souhaité lorsqu'ils ont achevé leurs études. L'abandon en cours de cycle est d'ailleurs une caractéristique forte de ce groupe, où les sorties en première année de $\mathrm{BEP}$ et en première professionnelle sont très fréquentes. Enfin, ce groupe de spécialités scolarise plutôt des jeunes d'origine urbaine, des enfants d'employés, et les descendants d'immigrés originaires du Maghreb s’y retrouvent en grand nombre.

Ce groupe de spécialités est le véritable « trou noir » de l'insertion. Les jeunes y ont sédimenté les handicaps tout au long de leurs parcours scolaires et ils éprouvent de façon homogène les plus grandes difficultés d’accès au marché du travail (Arrighi, Gasquet, Joseph, 2009b). Ils connaissent les plus forts taux de chômage trois ans après être sortis du système éducatif. En 2007, entre 27 et 37 \% d'entre eux sont au chômage, quand le taux pour l'ensemble des sortants de l'enseignement secondaire est de $21 \%$. Loin d'êtres ponctuelles, ces difficultés sont la trame de leurs trois premières années sur le marché du travail. Ils sont souvent hors de l'emploi : seulement $28 \%$ à $35 \%$ d'entre eux ont passé plus de deux ans et demi en emploi ${ }^{11}$ au cours de leurs trois premières années sur le marché du travail, ce qui est très peu comparé aux autres spécialités de formation ; à l'inverse, la part de ceux qui ont passé moins d'un an en emploi est très élevée, elle atteint même $40 \%$ parmi les sortants des formations du secrétariat, de l'habillement ou des services à la collectivité. De plus, lorsqu'ils occupent un emploi trois ans après la fin de leurs études, il s'agit souvent d'un emploi précaire : au mieux, la moitié d'entre eux sont recrutés sur un emploi à durée indéterminée. C'est le cas de 52 \% des jeunes formés en comptabilité qui, de ce point de vue, sont mieux lotis que ceux issus du secrétariat (47\%), de l'habillement (45 \%) et des spécialités de transformations (43\%). Au final, l'observation longitudinale de leurs trois premières années de vie active montre que seulement un cinquième des jeunes issus de ces spécialités a connu une stabilisation ${ }^{12}$ dans un emploi pérenne. Les individus formés dans les spécialités industrielles des transformations ont certes un accès à l'emploi relativement moins difficile (leur taux de chômage en 2007 est de 27 \%), mais ils n'occupent pas plus fréquemment que les autres jeunes du groupe un emploi à durée indéterminée. Enfin, ces jeunes sont le public privilégié des formes particulières d'emploi que sont les contrats aidés et l'intérim. Les emplois aidés concernent principalement les jeunes formés en habillement (27\% d'entre eux en ont connu au moins un au cours des trois ans), en comptabilité (27\%) et dans les services à la collectivité (17 \%). L'intérim est omniprésent dans les trajectoires des jeunes formés

\footnotetext{
11 À l'exception des jeunes formés dans une spécialité des industries de transformation qui sont $46 \%$ dans ce cas.

12 Avoir connu une période continue d'emploi de plus de 18 mois et occuper un emploi à durée indéterminée à la date de l'enquête (ou être employé comme fonctionnaire). Voir Arrighi, Gasquet, Joseph, 2009b.
} 
dans les spécialités des transformations (47\%) et important pour ceux issus de la comptabilité (28\%).

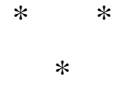

L'observation des trajectoires scolaires des jeunes de génération 2004 qui sont entrés dans la vie active à l'issue d'une formation de l'enseignement secondaire montre qu'ils se sont souvent heurtés ${ }^{13}$ à de profondes rigidités de l'offre éducative et que celles-ci ont conditionné leur orientation. Contrairement aux idées reçues, l'apprentissage apparaît le plus souvent comme un choix positif, et il en va de même des orientations conduisant aux métiers dits en tension. En revanche, les formations dont la pertinence est la moins reconnue sur le marché du travail, comme celles de la comptabilité ou du secrétariat (hors enseignement supérieur), celles des services à la collectivité, ou celles du textile et de l'habillement, sont également celles où la proportion de jeunes orientés par défaut est la plus importante. Entre 40 et $50 \%$ des jeunes qui les ont suivies ne les avaient pas choisies. Ces « orientations frustrées » sont le résultat d'un processus « d'affectation » destiné à optimiser le remplissage des classes d'un système éducatif rigidement partitionné en sous-systèmes sélectifs. Si la hiérarchie entre l'enseignement général, l'enseignement technologique et l'enseignement professionnel - bien que regrettée - est largement admise, celle qui partitionne l'enseignement professionnel est souvent ignorée dans l'analyse. Or, l'enseignement professionnel, et dans une moindre mesure l'enseignement technologique sont très cloisonnés, et la question qui se pose est celle de la distribution des élèves dans ces différents compartiments. Les régularités statistiques que nous constatons en observant la morphologie d'une cohorte de sortants du système éducatif ${ }^{14}$ montrent que celle-ci doit peu au hasard. Elle apparaît comme la résultante d'un univers de rigidités qui, au critère

\footnotetext{
133 jeunes sur 10 déclarent une orientation non conforme à leur premier vœu ; sachant que l'expression de ce premier vœu résulte déjà d'un arbitrage fait par le jeune entre ses aspirations premières et une liste fermée de possibilités qui lui sont proposées.

14 Rappelons ici que ces observations se fondent sur des données quantitatives et ne se placent à aucun moment sur le plan des biographies, plan qui concerne les conseillers d'orientation.
}

de l'excellence académique ${ }^{15}$ ajoute un faisceau de contraintes spécifiques : contraintes liées à la rareté relative des spécialités de formation sur un territoire donné ; contraintes liées à la nature fortement sexuée de ces formations ; contraintes liées à l'existence de discriminations ; contraintes liées à l'appétence des jeunes en lien avec leur histoire familiale ${ }^{16}$ et aux possibles de l'âge adolescent.

Dans la pratique, les choix sont souvent très limités. Ils ne se déploient que dans un nombre restreint d'établissements, regroupés dans les bassins de formation ${ }^{17}$, qui, pour des raisons inscrites dans la démographie et l'histoire locale ${ }^{18}$, offrent des gammes d'opportunités très diverses. De la plus riche à la plus frugale.

Dès lors, la portée d'une réforme de l'orientation scolaire apparaît limitée.

Non que celle-ci ne souffre de certains déficits qui sont connus et qu'il convient de corriger au plus grand bénéfice des élèves et des finances publiques (Haut Conseil de l’Éducation, 2008). Un consensus existe d'ailleurs aujourd'hui ${ }^{19}$ sur les principes d'une

\footnotetext{
${ }^{15}$ Excellence académique dont on sait, par ailleurs, qu'elle manifeste surtout les effets de l'origine sociale.

${ }^{16}$ Le paradoxe est d'ailleurs savoureux: c'est aux jeunes les moins biens armés pour la vie et les plus enclavés que s’adresse le plus précocement l'injonction du choix adulte, "l'injonction du projet ». Les autres auront eux le temps de butiner, d'hésiter et de mûrir en déroulant leur jeunesse dans le cocon des filières générales.

${ }^{17}$ Les bassins de formation forment un découpage fonctionnel du territoire par les services de l'Éducation nationale. Espaces géographiques de coopération et de mutualisation des établissements du second degré, ils sont le périmètre de définition de l'offre d'enseignement et de formation (carte scolaire, schéma des formations, carte des langues et des options, dispositifs d'insertion...). Une circulaire du 20 juin 2001 (BO n 26 du 28 juin 2001) précise le cadre général des missions et de l'organisation de ces bassins.

18 "On a vu tout au long de cette étude à quel point la logique "d'équité scolaire" qui indexe les processus d'orientation des jeunes à la performance scolaire avérée tendait à organiser les structures locales de formation en fonction de l'origine socioculturelle dominante des viviers présents sur la zone. Au point que l'on en vient à se demander quelle part peut jouer la relation de la spécialité de formation à l'emploi » (Caro, Hillau, 1997, p. 102).

19 Cette réorganisation peut prendre la forme d'un transfert ; c'est la solution que préconise le Haut Conseil de l'Éducation : «L'État devrait transférer au Régions, avec les moyens nécessaires, la responsabilité de l'accueil et de l'information en matière d'orientation des jeunes [...] La fonction de conseil en orientation, scolaire et professionnelle, devrait être maintenue au sein des établissements, mais elle ne peut plus être l'exclusivité d'un corps spécialisé de fonctionnaires; ceux qui seront appelés à exercer cette fonction devront avoir des compétences garantissant l'ouverture aux réalités sociales et professionnelles. Ces compétences seront répertoriées dans un référentiel qui reste à établir ». Bilan
} 
telle réforme. D'une part, insérer les Centre d'information et d'orientation (CIO) au sein d'un service public régional de l'orientation afin que ceux-ci puissent êtres intégrés dans la rationalisation du dispositif aujourd'hui complexe et redondant de l'Accueil Information Orientation. D'autre part, intégrer plus précisément le métier de conseiller d'orientation au sein des établissements scolaires et se donner les moyens de contrôler la qualité de son exercice.

Simplement, cette réforme nécessaire n'entame pas l'essentiel. Le dispositif d'orientation scolaire ne peut faire abstraction de sa fonction première : celle d'affecter les élèves et d'optimiser l'occupation des structures pédagogiques existantes («student distribution »). Quelle que puisse être sa qualité, il ne peut faire qu'avec ce qu'il a. C'est-à-dire avec, d'une part, des places limitées dans des dispositifs, soit rationnés (l'apprentissage), soit exigeants en compétences scolaires, jaloux de leur prestige et peu ouverts au métissage des savoirs (l'enseignement général), soit extrêmement coûteux en encadrement et en équipement technique (l'enseignement technologique et professionnel industriel). Et, d'autre part, des places disponibles dans des dispositifs soit périmés mais existants (les formations aux métiers du textile et de l'habillement), soit insuffisamment attractifs (les formations aux métiers de l'entretien et de la sécurité), soit peu coûteux mais inadéquats ${ }^{20}$ (secrétariat et

des résultats de l'École - 2008 - L'orientation scolaire op. cité (p. 35)... ou d'une intégration dans un grand service public national de l'orientation tout au long de la vie : centres d'information et d'orientation (CIO), services communs universitaires et interuniversitaires d'accueil, d'orientation et d'insertion professionnelle (SCUIO), Association nationale pour la formation professionnelle des adultes (AFPA)... dont l'action en région serait financée, pilotée et coordonnée avec les autres acteurs (Missions Locales, Permanence Accueil Information Orientation (PAIO), Fongecif (Fonds de gestion du congé individuel de formation), Maisons de l'information sur la formation et l'emploi (Mife), Agence nationale pour l'emploi (ANPE)), dans le cadre d'une coopération ÉtatRégion, selon des formes institutionnelles à expérimenter (Boras et alii, 2008).

${ }^{20}$ Les emplois cibles de ces formations (employés administratifs, aides-comptables, secrétaires) sont aujourd'hui très majoritairement comptabilité). Il est donc déraisonnablement optimiste d'imaginer qu'à elle seule une réforme de l'orientation scolaire éradique les "ghettos éducatifs » qui, à l'instar d'un tamis, rassemblent progressivement les jeunes dotés d'un triple handicap : une localisation spatiale pénalisante, un niveau scolaire médiocre ${ }^{21}$ et un réseau social défectueux. Pour éviter le pire d’une " sortie sans diplômes ", ils sont engagés dans un simulacre de poursuite d'études qui montre l'incapacité du modèle de la «méritocratie scolaire »22 à répondre efficacement à l'injonction d'élévation du niveau moyen d'éducation.

C'est toute l'architecture compartimentée et hiérarchisée de l'enseignement secondaire, en France, qu'il faudrait sans doute revoir pour que cesse enfin l'enfermement stigmatisant d'une fraction importante de la jeunesse. Mais, pour ce faire, il faudrait qu'un nouveau contrat lie la Nation à son École, qui ne soit pas exclusivement celui de "l'élitisme républicain » dont chaque jour qui passe démontre les limites. De nombreux chercheurs l'appellent de leurs vœux (Dubet, 2004 ; Duru-Bellat, 2006 ; Baudelot Establet, 2009).

Il est vrai que le chantier est ambitieux et autrement périlleux...

occupés par des diplômés du supérieur. D’ailleurs, les formations en alternance (en apprentissage ou en contrat de professionnalisation) qui préparent à ces fonctions recrutent exclusivement des bacheliers.

21 À l'exception des spécialités des services à la collectivité, ces spécialités ne scolarisent pas particulièrement les jeunes les plus en difficulté au collège. En effet, les élèves qui ne sont pas issus d'une troisième générale (troisième technologique, troisième d'insertion, classes préparatoires à l'apprentissage (CPA)) sont en proportion beaucoup plus nombreux dans les formations des spécialités conduisant aux métiers de proximité (bâtiment, alimentation, bois, etc.). C'est donc la conjonction d'une localisation spatiale, d'un niveau scolaire un peu insuffisant et d'un réseau social inefficace qui « oriente » vers ces impasses.

22 Selon la formule de Christian Baudelot et Roger Establet dans L'élitisme républicain: l'école française à l'épreuve des comparaisons internationales, Paris, Seuil, 2009, Collection « La République des idées ». 


\section{Bibliographie}

Arrighi J.-J., Gasquet C., Joseph O. (2009a), «Qui sort de l'enseignement secondaire? Origine sociale, parcours scolaires et orientation des jeunes de la Génération 2004 », Céreq, Note Emploi Formation $n^{\circ} 41$, juillet.

Arrighi J.-J., Gasquet C., Joseph O. (2009), «L'insertion des sortants de l'enseignement secondaire » Céreq, Note Emploi Formation n 42, juillet.

Baudolot Ch., Establet R. (2009), L'élitisme républicain: l'école française à l'épreuve des comparaisons internationales. Paris, Seuil, Collection « La République des idées ».

Berthet Th., Dechezelles S., Gouin R., Simon V. (2008), « Orientation : la parole aux élèves », Céreq, Note Emploi Formation $n^{\circ}$ 34, septembre.

Borras I. (2008), « Le pilotage de l'orientation tout au long de la vie : le sens des réformes », Cereq, Note Emploi Formation $n^{\circ} 29$, juillet.

Boudesseul G., Grelet Y. (2008), «Choix d'orientation et logiques institutionnelles », Céreq, Note Emploi Formation n ${ }^{\circ}$ 32, août.

Caro P., Hillau B. (1997), « La logique dominante des publics scolaires. Offre de formation et environnement local ", Formation Emploi, n ${ }^{\circ}$ 59, juilletseptembre.
Dubet F. (2004), L'école des chances: qu'est-ce qu'une école juste?, Paris, Seuil, Collection « La République des idées ».

Duru-Bellat M. (2006), L'inflation scolaire. Les désillusions de la méritocratie, Paris, Seuil, Collection « La République des idées ».

Farvaque N. (2009), « Discriminations dans l'accès au stage : du ressenti des élèves à l'intervention des enseignants ", Formation Emploi $\mathrm{n}^{\circ} 105$, janviermars, pp. 21-36.

Grelet Y., Viney X. (1991), «Dix ans d'insertion professionnelle des jeunes à l'issue de l'enseignement technique court (CAP-BEP », Paris, Céreq, Collection des études, $\mathrm{n}^{\circ} 58$.

Grelet Y. (2005), «Enseignement professionnel, spécialité de formation et reproduction sociale », Éducation et Formation $\mathrm{n}^{\circ}$ 72, septembre.

Haut Conseil de l'Éducation (2008), Bilan des résultats de l'École - 2008-L'orientation scolaire, rapport téléchargeable sur http://www.hce.education.fr

Moreau G. (2008a), «Apprentissage : une singulière métamorphose », Formation Emploi, n 101, pp. 119-133, janvier-mars.

Moreau Gilles (sous la dir.) (2008b), Les apprentis ligériens en 2006/2007. Enquête sociologique, rapport au conseil régional des Pays de la Loire, juillet. 
Résumé

\title{
Orientation et affectation : la sélection dans l'enseignement professionnel du second degré
}

\author{
Jean-Jacques Arrighi et Céline Gasquet
}

Observant les caractéristiques et les scolarités des jeunes qui entrent dans la vie active à l'issue de l'enseignement secondaire, cet article montre que leur orientation doit peu au hasard. À partir de l'enquête Génération 2004 du Céreq, il remet en cause les idées communément admises à l'égard des jeunes : leur réticence vis-à-vis de l'apprentissage et leur appétence pour des spécialités sans débouchés. II rappelle l'importance de rigidités trop souvent négligées comme les discriminations à l'œuvre au sein de l'apprentissage et la ségrégation sexuée des spécialités de formation. Prolongeant la hiérarchie tacite de l'enseignement - général, technologique puis professionnel -, les spécialités de formation et les filières organisent un système lui-même hiérarchisé où les jeunes les moins bien informés ou dotés se trouvent relégués dans des formations sans perspectives professionnelles très claires. Dans ce contexte, l'article invite à s'interroger sur la portée d'une réforme de l'orientation qui ne concernerait que son organisation.

\section{Mots clés}

Orientation scolaire-professionnelle, apprentissage, enseignement technique-professionnel, enseignement secondaire

Journal of Economic Literature: I 21, J 24 